\title{
Low plasma CC16 levels in smokers are associated with a higher risk for chronic bronchitis
}

\begin{abstract}
To the Editor:
Club cell protein (CC16 in humans) plays a crucial role in maintaining homeostasis of the airway epithelium [1] and has anti-inflammatory activity in the lung [2]. Serum CC16 levels are low in cigarette smokers [3] and increase following smoking cessation [4], but patients with chronic obstructive pulmonary disease (COPD) show progressive decrease in CC16 with disease severity [5]. Thus, although the association between circulating levels of $\mathrm{CC} 16$ and airflow obstruction is accepted, the association between circulating CC16 and chronic bronchitis, and whether a link in association exists between circulating CC16 levels, lung function decline and chronic bronchitis have not been shown. Therefore, we tested the hypothesis that circulating levels of $\mathrm{CC} 16$ are associated with the presence of chronic bronchitis in ever smokers.
\end{abstract}

For these studies, we used data from two cohorts of smokers. Detailed description of the prospective cohort of current and former smokers in the Lovelace Smokers Cohort (LSC) (median follow-up 6 years) has been published previously $[6,7]$. The ECLIPSE cohort of COPD patients has been extensively described $[8,9]$. Chronic bronchitis is defined as a self-reported cough productive of phlegm for at least 3 months per year for 2 or more consecutive years. Comparisons of plasma CC16 levels in smokers with chronic bronchitis versus those without chronic bronchitis in the LSC and ECLIPSE cohorts, and rapid forced expiratory volume in $1 \mathrm{~s}$ (FEV1) decliners versus non-decliners in the LSC, were performed using SAS 9.3 (Cary, NC, USA).

The association between plasma CC16 levels and $\mathrm{CB}$ was assessed in subgroups within the two cohorts with a minimum follow-up period of 3 years. Among the 2273 participants enrolled in the LSC, 1210 subjects with a minimum of 3 years of observation were selected for analysis of plasma CC16 levels. Commercially available ELISA kits were used for the CC16 measurement (BioVendor Research and Diagnostic Products, Asheville, NC, USA) according to manufacturer's instructions. p-values for univariate comparisons were calculated using paired t-tests or Mann-Whitney rank sum tests. Jonckheere-Terpstra tests or ANOVAs were performed in comparisons of multiple ordered alternatives. Chronic bronchitis subjects had significantly lower CC16 levels (chronic bronchitis $2.7 \mathrm{ng} \cdot \mathrm{mL}^{-1}$, $95 \% \mathrm{CI} 0.9-7.3$; versus no chronic bronchitis $3.4 \mathrm{ng} \cdot \mathrm{mL}^{-1}$, 95\% CI 1.1-9.0) Multivariable logistic regression models adjusted for age, sex, baseline body mass index (BMI), pack years of smoking, baseline current smoking status, COPD and rapid decline status showed that low $\mathrm{CC} 16$ levels are associated with higher rates of baseline chronic bronchitis (OR 0.73, $\mathrm{p}=0.013$ ) independent of concomitant COPD and lung function decline. To limit over-adjustment, per cent predicted FEV1 was omitted as covariate in these models. This finding was validated in a multivariable analysis in the ECLIPSE cohort adjusted for the same covariates showing that lower CC16 levels were associated with higher rates of chronic bronchitis (OR 0.71, p=0.002). As in earlier findings that low plasma CC16 levels are associated with increased FEV1 decline in COPD $[8,10]$, we also found that low CC16 is associated with rapid decline in FEV1 in ever smokers. In a logistic regression model, adjusted for age, sex, Hispanic ethnicity, baseline BMI, pack years, current smoking at baseline and baseline per cent predicted $\mathrm{FEV} 1, \mathrm{CC} 16$ showed a negative association with COPD (OR 0.71, p=0.01; $\mathrm{n}=313 / 1210$ ). This is also consistent with our recent findings in mice and humans [5].

This study shows that in two large cohorts higher plasma CC16 levels are associated with lower frequencies of chronic bronchitis and that chronic bronchitis was associated with lower plasma CC16 levels. Clearly baseline lung function is also strongly associated with CC16 levels, more so in fact than chronic bronchitis in the older, sicker ECLIPSE cohort, indicating that CC16 is commensurate with existing lung damage. Indeed, the findings that younger smokers and current smokers have lower CC16 levels together with published data showing CC16 is associated with early mortality [11] suggest that low CC16 may serve as a predictor of future lung problems.

To determine whether the low levels of circulating CC16 may be caused by genetic variants, we selected an independent group of 1196 subjects for analysing 10 tag single nucleotide polymorphisms (SNPs) present in the promoter region of CC16. The gene encoding for CC16 is SCGB1A1. We used 1193 subjects for whom plasma CC16 and SNP analysis data were available to determine the association of 10 SNPs with plasma CC16 levels (370 with and 823 without chronic bronchitis). An observed trend toward association 
of plasma CC16 levels with chronic bronchitis led to the additional genotyping in 668 additional subjects for rs72923298 to provide data for a total of 1864 subjects. Plasma samples were drawn at baseline examination. Common SNPs $(n=10$, minor allele frequency $\geqslant 0.05)$ located within the $20 \mathrm{~kb}$ upstream of CC16 gene were genotyped in 1196 LSC subjects using the HumanOmni2.5-4v1-H BeadChip (Illumina, San Diego, CA, USA). These 10 SNPs, each having pairwise $r^{2}<0.8$, were included in the genetic association analyses. All 10 SNPs were associated with altered plasma CC16 levels. Among these 10 SNPs, two SNPs (rs3741240 and rs2509963) were associated with rapid decline and one of the alleles associated with increased plasma CC16 levels was also associated with reduced risk for rapid FEV1 decline. A different SNP (rs72923298) was significantly associated with plasma CC16 levels (OR 1.12, p=0.049) and negatively associated with chronic bronchitis (OR 0.75, $\mathrm{p}=0.05$ ). Although the available sample size is unable to support adjustment for multiple comparisons, the SNP exhibits a strong association with circulating CC16 levels. Studies in additional cohorts are planned.

Potential interactions between rapid FEV1 decline status and chronic bronchitis initially were explored in univariate analyses. Comparing subjects with normal FEV1 decline and no chronic bronchitis, chronic bronchitis only, rapid decline only, and both rapid decline and chronic bronchitis, showed significant differences between these groups for plasma CC16 levels. Subsequent adjusted multivariate analyses confirmed that the main effects of rapid FEV1 decline and chronic bronchitis maintain significant associations with plasma CC16 levels (point estimate (PE) -0.1 (SE 0.01), $\mathrm{p}=0.01$; PE -0.1 (SE 0.1), p=0.02, respectively). However, neither of the interactions between rapid decline and chronic bronchitis or COPD and chronic bronchitis were significantly associated with plasma CC16 levels (PE 0.02 (SE 0.1), p=0.8; and PE 0.1 (sE 0.1), $\mathrm{p}=0.2$, respectively) (table 1). These findings suggest that CC16 may affect independent pathways for FEV1 decline and chronic bronchitis. Further support that CC16 may employ different mechanisms to affect decline in FEV1 and chronic bronchitis stems from the associations with SNPs in the CC16 promoter. Two SNPs among 10 candidates in the promoter region of the CC16 gene were associated with plasma CC16 levels and risk for rapid FEV1 decline status. However, a different SNP was associated both with plasma CC16 levels and chronic bronchitis. Various isoforms of CC16 are produced in the airways [12], and future studies need to clarify whether they differ in their functions in protecting from FEV1 decline and chronic bronchitis.

Collectively, our observations support a biological role for CC16 in the development of chronic bronchitis independent of sex. First, the LSC consists primarily of women and our results confirm those from studies performed in predominantly male cohorts. Therefore, the beneficial effects of CC16 in ever smokers are unlikely to be gender specific. Secondly, even after adjustment in multivariable models for age, sex, baseline BMI, pack years of smoking and baseline current smoking status, the observed effect not only persists but is strengthened. These observed associations are consistent with published results wherein CC16 has been associated with anti-inflammatory activity in lungs $[2,13]$ and with regression of bronchial dysplasia in former smokers [14]. Taken together, these studies support an integral role for this protein in lung health.

TABLE 1 Multivariate analysis of the association of plasma CC16 levels with chronic bronchitis, rapid decline in forced expiratory volume in $1 \mathrm{~s}$ (FEV1) and their interaction

\begin{tabular}{lcc} 
& PE (sE) & p-value \\
\hline Age & $0.018(0.002)$ & $<0.0001$ \\
Female & $-0.249(0.042)$ & $<0.0001$ \\
Hispanic & $-0.026(0.048)$ & 0.589 \\
Body mass index & $0.002(0.003)$ & 0.430 \\
Pack years & $-0.001(0.001)$ & 0.106 \\
Baseline smoker & $-0.328(0.040)$ & $<0.0001$ \\
Rapid decline & $-0.112(0.045)$ & 0.012 \\
Chronic bronchitis & $-0.134(0.055)$ & 0.015 \\
COPD & $-0.245(0.054)$ & $<0.0001$ \\
Rapid decline + chronic bronchitis & $0.015(0.078)$ & 0.849 \\
Chronic bronchitis + COPD & $0.108(0.085)$ & 0.203 \\
\hline
\end{tabular}

Data are presented as point estimates with standard errors. Multivariable linear regression adjusted for age, sex, Hispanic ethnicity, baseline body mass index, pack years of smoking, smoking status at baseline, rapid FEV1 decline status, baseline chronic bronchitis and the interaction of baseline chronic bronchitis and rapid FEV1 decline status. COPD: chronic obstructive pulmonary disease. 
@ERSpublications

Plasma CC16 levels are associated with CC16 gene polymorphisms and inversely with chronic bronchitis http://ow.ly/O8CHk

Hans Petersen ${ }^{1}$, Shuguang Leng ${ }^{1}$, Steven A. Belinsky ${ }^{1}$, Bruce E. Miller ${ }^{2}$, Ruth Tal-Singer ${ }^{2}$, Caroline A. Owen ${ }^{3}$, Bartolome Celli ${ }^{3}$ and Yohannes Tesfaigzi ${ }^{1}$

${ }^{1}$ Lovelace Respiratory Research Institute, Albuquerque, NM, USA. ${ }^{2}$ Research and Development, GSK, King of Prussia, PA, USA. ${ }^{3}$ Pulmonary Division, Brigham and Women's Hospital, Harvard Medical School, Boston, MA, USA.

Correspondence: Yohannes Tesfaigzi, COPD Program, Lovelace Respiratory Research Institute, 9495 Ridgecrest Dr., SE, Albuquerque, NM 87108, USA. E-mail: ytesfaig@lrri.org

Received: March 222015 | Accepted after revision: May 252015 | First published online: July 092015

Support statement: This work was supported by the State of New Mexico (appropriation from the Tobacco Settlement Fund), from the National Institutes of Health (P50HL107165 for B. Celli and Y. Tesfaigzi, RO1 HL068111 and ES015482 to Y. Tesfaigzi, R01CA164782 for Y. Tesfaigzi/S.A. Belinsky, R01 CA097356 for S.A. Belinsky, HL105339, HL114501, AI111475 for C.A. Owen, FAMRI CIA 123046 for C.A. Owen) and funds from GSK. Funding information for this article has been deposited with FundRef.

Conflict of interest: Disclosures can be found alongside the online version of this article at erj.ersjournals.com

\section{References}

$1 \quad$ Stripp BR, Reynolds SD, Boe IM, et al. Clara cell secretory protein deficiency alters Clara cell secretory apparatus and the protein composition of airway lining fluid. Am J Respir Cell Mol Biol 2002; 27: 170-178.

2 Long $\mathrm{XB}, \mathrm{Hu} \mathrm{S}$, Wang $\mathrm{N}$, et al. Clara cell $10-\mathrm{kDa}$ protein gene transfection inhibits NF-kappaB activity in airway epithelial cells. PloS One 2012; 7: e35960.

3 Bernard AM, Roels HA, Buchet JP, et al. Serum Clara cell protein: an indicator of bronchial cell dysfunction caused by tobacco smoking. Environ Res 1994; 66: 96-104

4 Chen J, Lam S, Pilon A, et al. The association between the anti-inflammatory protein CC10 and smoking status among participants in a chemoprevention trial. Cancer Epidemiol Biomarkers Prev 2007; 16: 577-583.

5 Laucho-Contreras ME, Polverino F, Gupta K, et al. Protective role for club cell secretory protein-16 (CC16) in the development of chronic obstructive pulmonary disease. Eur Respir J 2015; 45: 1544-1556.

6 Sood A, Petersen H, Blanchette CM, et al. Wood smoke exposure and gene promoter methylation are associated with increased risk for COPD in smokers. Am I Respir Crit Care Med 2010; 182: 1098-1104.

7 Hunninghake GM, Cho MH, Tesfaigzi Y, et al. MMP12, lung function, and COPD in high-risk populations. N Engl J Med 2009; 361: 2599-2608.

8 Vestbo J, Edwards LD, Scanlon PD, et al. Changes in forced expiratory volume in 1 second over time in COPD. N Engl J Med 2011; 365: 1184-1192.

9 Kim DK, Cho MH, Hersh CP, et al. Genome-wide association analysis of blood biomarkers in chronic obstructive pulmonary disease. Am J Respir Crit Care Med 2012; 186: 1238-1247.

10 Lomas DA, Silverman EK, Edwards LD, et al. Evaluation of serum CC-16 as a biomarker for COPD in the ECLIPSE cohort. Thorax 2008; 63: 1058-1063.

11 Guerra S, Vasquez MM, Spangenberg A, et al. Serum concentrations of club cell secretory protein (Clara) and cancer mortality in adults: a population-based, prospective cohort study. Lancet Respir Med 2013; 1: 779-785.

12 Broeckaert F, Clippe A, Wattiez R, et al. Lung hyperpermeability, Clara-cell secretory potein (CC16), and susceptibility to ozone of five inbred strains of mice. Inhal Toxicol 2003; 15: 1209-1230.

13 Dodge DE, Plopper CG, Rucker RB. Regulation of Clara cell $10 \mathrm{kD}$ protein secretion by pilocarpine: quantitative comparison of nonciliated cells in rat bronchi and bronchioles based on laser scanning confocal microscopy. Am J Respir Cell Mol Biol 1994; 10: 259-270.

14 Chen J, Lam S, Pilon A, et al. Higher levels of the anti-inflammatory protein CC10 are associated with improvement in bronchial dysplasia and sputum cytometric assessment in individuals at high risk for lung cancer. Clin Cancer Res 2008; 14: 1590-1597.

\section{Mefloquine as a potential drug against multidrug-resistant tuberculosis}

To the Editor:

In the article by ALSAAD et al. [1] in a recent issue of the European Respiratory Journal the authors reviewed six drugs with antimicrobial activity against Mycobacterium tuberculosis (phenothiazine, metronidazole, 